\title{
Implementación de una innovación docente en la asignatura "Terapéutica Enfermera, Alimentación y Cuidados"
}

RUBÉN MORILLA ROMERO DE LA OSA

Universidad de Sevilla

Departamento de Enfermería

morilla2@us.es

ORCID: https://orcid.org/0000-0002-0717-3938

D.O.I.: http://dx.doi.org/10.12795/JDU.2018.i01.26

Pp.: 466-481

\section{Resumen}

Se presenta el primer ciclo de mejora llevado a cabo en la asignatura "Terapéutica Enfermera, Alimentación y Cuidados" de 2o curso del Grado en Enfermería, en la unidad docente Virgen del Rocío, con 51 estudiantes matriculados en un grupo grande, donde se planificó una secuencia de actividades que propiciaron la participación de estos en su proceso de aprendizaje. Se realizó un análisis previo y final de conocimientos, mediante un cuestionario ad hoc de 13 preguntas cerradas con cuatro opciones de respuesta, y en todas las preguntas hubo una mejora en los conocimientos. 
Palabras clave: Alimentación y Cuidados, Docencia Universitaria, Enfermería, Experimentación Docente Universitaria, Terapéutica Enfermera.

\section{Breve descripción del contexto de la intervención}

El ciclo de mejora (CM) ha constado de diez horas repartidas en cinco clases de dos horas cada una y se ha realizado en la asignatura de 2 - curso del Grado en Enfermería (2o cuatrimestre): "Terapéutica Enfermera, Alimentación y Cuidados" (TEAC), cuyo carácter en el plan de estudio del grado en Enfermería se corresponde con la formación básica y posee una carga lectiva de 6 créditos ECTS. Está dividida en cuatro grupos grandes repartidos por las diferentes unidades docentes de la Facultad de Enfermería, Fisioterapia y Podología de la Universidad de Sevilla: dos en Perdigones, uno en Virgen de Valme y otro en del Rocío.

El grupo sobre el que se ha llevado cabo la intervención es el de Virgen del Rocío, que en el presente curso contaba con 51 estudiantes matriculados, de los que 11 $(21,5 \%)$ son hombres, siendo esta una proporción mayor a la observada en cursos anteriores. Las clases se imparten en un aula del centro docente Virgen del Rocío, esta tiene un tamaño adecuado para acoger al grupo, y una buena ventilación, climatización e iluminación, además, posee ordenador y proyector. Aunque las bancas de los alumnos están todas al mismo nivel y fijadas al suelo, lo que impide sus desplazamientos o reubicaciones.

La asistencia a clase, en los grupos grandes donde se imparten clases teóricas, no es obligatoria, por lo que alumnos repetidores o trabajadores no suelen venir; no obstante, la asistencia suele ser elevada, exceptuado contadas ocasiones como días que coinciden con evaluaciones de otras asignaturas. Estas clases suelen desarrollarse de forma expositiva por parte del profesorado, siendo estas el objeto de las intervenciones desarrolladas. El objetivo de esta experimentación es cambiar la metodología 
empleada en las clases teóricas de gran grupo, para que sean más dinámicas y participativas.

\section{Diseño del CM}

\section{Mapa de contenidos}

Debido a la forma que tiene de trabajar nuestra mente, estableciendo interconexiones entre conceptos, procedimientos y actitudes y sin dejar a un lado el hecho de que el conocimiento no se genera de forma aislada, sino en el seno de determinadas circunstancias históricas o sociales (Porlán, 2017), cada profesor tiene una visualización mental de los contenidos que desea impartir en la asignatura que imparte. Esta visualización puede ser jerárquica o no, y puede establecer infinidad de interconexiones entre los elementos que la forman que permiten explorar los contenidos de una forma dinámica, pero estructura. Por ello, la elaboración de mapas de contenidos se considera una actividad de buenas prácticas docente que mejoran la docencia universitaria (González, 2010).

En nuestro caso, se ha diseñado un mapa de contenidos no jerárquico, y que muestra como del acrónimo de la asignatura parten tres ramas, de las cuales dos, en amarillo, no han sido sujeto de intervención. Los contenidos trabajados en el presente CM están incluidos en rama descendente que es la principal de la asignatura (figura 1).

Jornadas de Formación e Innovación Docente del Profesorado | № 1 (2018) Esta obra se distribuye con la licencia Creative Commons 


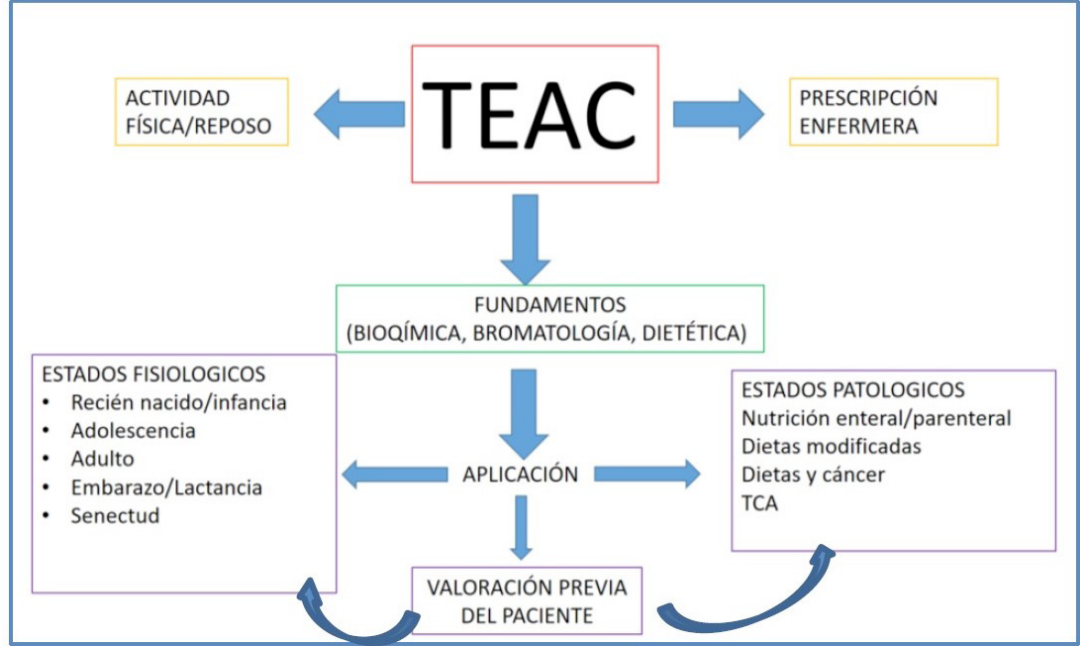

Figura 1. Mapa conceptual de la asignatura TEAC.

En cuanto a los contenidos conceptuales se han trabajo desde el punto de vista fisiológico los mecanismos que regulan las sensaciones de apetito y saciedad, así como los conceptos de metabolismo basal y factores que lo determinan o modifican. Este tipo de factores forman parte de los aspectos a valorar en un paciente y por tanto existe interrelación entre dichos contenidos. Por otro lado, los problemas de salud de las personas en ocasiones requieren modificaciones en sus dietas (dietas modificadas en contenido calórico, en hidratos de carbonos, grasas o proteínas), lo cual requiere también una valoración previa. A su vez todo ello se sustenta sobre conocimientos de bioquímica de los alimentos y de la nutrición, bromatología y elaboración de dietas.

También se desarrollaron contenidos procedimentales puesto que la valoración del paciente incluye la recogida sistemática de información clínica y sobre estilos de vida. Además, posterior instauración de la dieta requiere un análisis cualitativo (variedad de alimentos saludables) y cuantitativo (número y distribución de calorías). Finalmente se abordaron contenidos actitudinales, puesto que 
los alumnos deben aprender a tratar al paciente con respeto y empatía, y ser consciente de la confidencialidad que requiere la gestión de la información que en el personal sanitario depositan.

\section{Modelo metodológico y secuencia de actividades.}

Se parte de un modelo tradicional de transmisión del conocimiento unidireccional (García, 2000a). Sin embargo, asumiendo que los procesos de aprendizajes se sustentan en prácticas de estudios llevadas a cabo por el alumno y organizadas por el profesor (Cardelli, 2004), el modelo tradicional parece poco acertado en la actualidad. Por otro lado, nuestro modelo visualiza al estudiante universitario como una persona adulta, con capacidad de trabajo autónomo y con interés, desde una perspectiva personal, en aprender unos contenidos que les permita desarrollar una profesión en el futuro (Giné, 2008).

En base a estas premisas se planificó introducir una secuencia de actividades que propiciaran la participación del alumno en su propio aprendizaje. Además, la experimentación fue también un intento de relativizar la importancia de los contenidos centrados en los conceptos meramente teóricos y dar mayor protagonismo a contenidos procedimentales y actitudinales (aunque, como se indicó al inicio, estos se trabajan en los seminarios también). Todo ello supone, como se ha comentado anteriormente, un primer paso de metamorfosis hacia un modelo investigativo caracterizado por un nuevo papel del profesor. Este actúa más como dinamizador de la clase, en vez de como director y emisor de información (García, 2000b).

La secuencia de actividades quedó diseñada como sigue:

- Actividades previas a la clase: El profesor prepara el material de la unidad que corresponde y se lo envía a 4 miembros del alumnado previamente seleccionados ("alumnas o alumnos tutoras o tutores", voluntarias o 
voluntarios) con las indicaciones oportunas sobre qué contenidos deben preparar.

\section{- Actividades durante la clase:}

1. Se ofrecen cuatro voluntarias nuevas (miembros del alumnado que exponen) y se emparejan con los cuatro miembros del alumnado tutores. Salen de la clase durante diez minutos para comunicarse los contenidos y preparar la exposición.

2. En paralelo, el profesor introduce el tema mediante la exposición de situaciones reales y datos epidemiológicos que enmarcan la situación en nuestro contexto social actual.

3. Las parejas entran en clase y se exponen los contenidos. En caso de confusión u olvido por parte del alumnado que expone, o bien ante preguntas de otros estudiantes que éstos no son capaces de responder, los miembros del alumnado que tutorizan pueden intervenir. Si hay alguna pregunta que no saben responder, el profesor mediará la respuesta intentado primero que las participantes la descubran a partir de contenidos de otras asignaturas o temas relacionados.

4. El profesor hace un resumen integrador y aporta la información más compleja sobre estudios recientemente publicados, o sobre contenidos que no repartió entre los grupos.

5. Cuestiones y debate de clausura. Los alumnos y alumnas exponen dudas y experiencias sobre los contenidos expuestos y sobre casos que han conocido en su entorno personal o durante las prácticas clínicas.

Preguntas del cuestionario de conocimientos: A continuación, se muestran las preguntas del cuestionario de evaluación inicial y final.

\section{La tasa de metabolismo basal puede verse afec- tada por (señala la incorrecta):}


a) Estados que cursan con fiebre alta.

b) La neutralidad térmica.

c) Las situaciones estresantes.

d) La menstruación y los cambios hormonales

2. Qué característica diferencia una alergia alimentaria de una intolerancia o intoxicación alimentaria?

a) La duración de los síntomas es mayor en la alergia alimentaria que en la intoxicación o la intolerancia alimentaria.

b) La alergia alimentaria es una reacción adversa de mayor gravedad que intoxicaciones e intolerancias alimentarias.

c) La alergia alimentaria tiene su origen en una disfunción del sistema inmunológico, en la intoxicación y la intolerancia alimentaria el sistema inmunológico no está comprometido.

d) Ninguna de las anteriores es cierta.

3. Cual de las afirmaciones en relación con los alimentos que tomamos es falsa

a) Encontraremos ácidos grasos polinsaturados en el pescado azul

b) Leche y huevos tiene proteínas de escaso valor biológico

c) El aceite de oliva es rico en ácido graso monoinsaturado

d) frutas y verduras son ricas en vitaminas

4. Las últimas recomendaciones nutricionales indican (señale la incorrecta)

a) Disminuir las grasas animales

b) Aumentar las harinas integrales

c) Disminuir el azúcar y los dulces

d) Disminuir la leche y sus derivados

5. Consideramos causas de la desnutrición hospitalaria todas las siguientes excepto:

a) Disminución de ingresos

b) Disminución de consumo

Jornadas de Formación e Innovación Docente del Profesorado I № 1 (2018)

Esta obra se distribuye con la licencia Creative Commons Reconocimiento-NoComercial-SinObraDerivada $\quad 4.0$ Internacional (CC BY-NC-ND 4.0.) 
c) Aumento del consumo

d) Incremento de pérdidas

6. La energía que debe aportar la dieta de un diabético debe ser:

a) Hipocalórica.

b) Igual que la de un adulto sano normal.

c) Hipercalórica.

d) Menor que en individuos sanos

7. Como se comporta el organismo a medida que aumentamos en edad

a) Disminuye el metabolismo basal

b) Disminuye la inmunidad

c) disminuye la masa magra

d) Todas son correctas

8. Para reducir la ingesta de ácidos grasos saturados habrá que limitar la ingesta de:

a) Grasa en carne.

b) Aceite de oliva.

c) Aceite de coco.

d) a y c son correctas.

9. En la Pancreatitis crónica se prescribirá una dieta:

a) Dieta pobre en fibra.

b) Dieta rica en fibra.

c) Dieta pobre en grasas.

d) Dieta hipercalórica.

10. De las propiedades de la fibra dietética y sus efectos fisiológicos, señale la respuesta falsa:

a) Disminuye el tiempo de contacto, por lo que previene el cáncer de colon.

b) Aumenta el bolo fecal por lo que disminuye el estreñimiento.

c) Aumenta la absorción de nutrientes, como el hierro por lo que es beneficioso en anemias.

d) Disminuye el colesterol sanguíneo, por lo que previene y mejora enfermedades cardiovasculares.

11. Una dieta es equilibrada cuando incluye:

a) Un $30-32 \%$ de HC, un $10-12 \%$ de G, y un $55-60 \%$ de P. 
b) Un $55-60 \%$ de $\mathrm{HC}$, un $10-12 \%$ de $\mathrm{G}$, y un $30-32 \%$ de $\mathrm{P}$.

c) Un $40 \%$ de $\mathrm{HC}$, un $15-20 \%$ de G, y un $40 \%$ de P.

d) Un $55-60 \%$ de $\mathrm{HC}$, un $30-32 \%$ de $\mathrm{G}$, y un $10-12 \%$

12. Cuál es el parámetro más extendido para la evaluación del estado nutricional?

a) El peso.

b) La altura partida por el peso al cuadrado.

c) El peso partido por la altura al cuadrado.

d) El peso partido por la altura.

13. Una ración o intercambio equivale a:

a) 10 gr. de Hidratos de Carbono, 10gr. de Grasas, y 10 gr. de Proteínas.

b) $10 \mathrm{mg}$. de Hidratos de Carbono, $10 \mathrm{mg}$. de Grasas, y $10 \mathrm{mg}$. de Proteínas.

c) 1 gr. de Hidratos de Carbono, 1 gr. de Grasas, y 1 gr. de Proteínas.

d) 100 gr. de Hidratos de Carbono, 100 gr. de Grasas, y 100 gr. de Proteínas.

\section{Aplicación del CM}

\section{Relato Resumido de las sesiones.}

En general, el desarrollo de las sesiones ha sido más participativo que en las clases expositivas habituales. Los alumnos parecían algo más relajados, aunque no por ello dispersos, posiblemente porque la introducción de novedades elimina la rutina ayudando a focalizar en lo que se está haciendo. Asimismo, las alumnas tutoras solicitaron salir de la clase con portátiles porque incluso habían diseñado un power point para la exposición y las alumnas que exponían usaron estas diapositivas en vez de las previstas en clase. Esto supuso un avance novedoso, puesto que se trabajó sobre las diapositivas elaboradas por estudiantes y las elaboradas por el profesor (actividades 3 
y 4 respectivamente) aportando visiones diferentes de los mismos contenidos. Posiblemente esta dinámica ayuda a fijar los contenidos puesto que se ven de diferentes formas, tanto gráficamente como oralmente. El alumnado que no formó parte activa de la experimentación (ni exponiendo ni tutorizando) participó también con frecuencia, y lo hicieron fundamentalmente de dos formas:

1. Preguntando dudas y/o curiosidades, que fueron resueltas siguiendo la escala inversa de informadores (primero alumnas que exponen, segundo tutoras y por último el profesor).

2. Aportando experiencias personales o vividas en las prácticas clínicas, lo cual sirvió para visualizar lo actual e importante, profesionalmente hablando, que es el tema.

Al finalizar se percibía un sentimiento generalizado de satisfacción por parte del alumnado, manifestado principalmente por la actitud participativa del estudiantado en el debate de clausura, en el que, además de preguntas, expusieron experiencias personales usando los contenidos abordados. Por lo demás, los resultados del test de satisfacción (ver apartado de evaluación del diseño e identificación de mejoras) constatan lo percibido.

\section{Evaluación de los aprendizajes de los alumnos.}

Para llevar a cabo la evaluación de los aprendizajes de los alumnos se cumplimentó de forma voluntaria el test de 13 preguntas que se han mostrado anteriormente antes y después de llevar a cabo el CM.

Como resultados globales podemos decir que el promedio en el pretest fue de 7,4 sobre 13 lo que implica una proporción de aciertos del 56,9\%. Una vez llevada a cabo la intervención, el número promedio de aciertos subió a 10,6 aciertos sobre 13, lo que supone una proporción de aciertos del 81,5\%. Estos datos nos muestran un incremento positivo en la proporción media de aciertos, lo que muestra 
de forma cuantitativa la efectividad de la metodología empleada en el aprendizaje. No obstante, no deberíamos quedarnos simplemente en una evaluación global, siendo de sumo interés el análisis de la evolución del aprendizaje por pregunta (figura 2) a fin de detectar áreas de mejora.

Se percibió una mejora en todas las preguntas, siendo las preguntas 8,11 y 13 las que más incrementaron en número de respuestas correctas entre ambos test. La gráfica revela también que en las preguntas 1, 2, 4, 7 y 10 el incremento de respuesta correctas no es elevado, pero porque de partida ya se percibe una alta proporción de aciertos por lo que, teniendo en cuenta que hablamos de más de un tercio de preguntas (38,5\%) podemos considerar que los alumnos tienen un buen nivel de partida en cuanto a conocimientos de base de la asignatura.

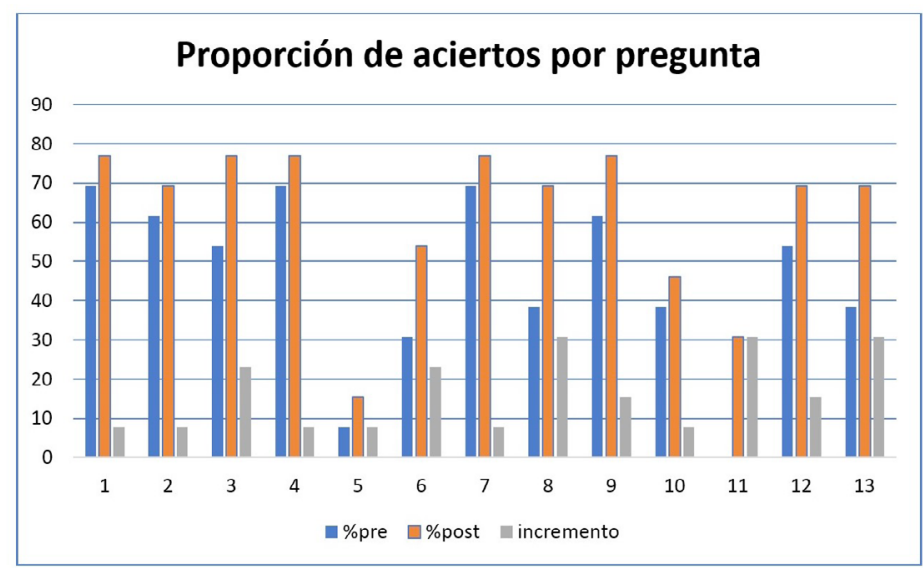

Figura 2. Análisis de evolución del aprendizaje por pregunta. Elaboración propia

Figura 2. Análisis de evolución del aprendizaje por pregunta. Elaboración propia

Por otro lado, la figura 1 expuesta anteriormente, también nos sirve para identificar áreas de mejora puesto que las preguntas 5 y 10, a pesar de incrementar el número de aciertos, la diferencia entre pretest y postest no es muy significativa, quedando, además la proporción de aciertos por debajo del 20 y el 50\% respectivamente. 


\section{Evaluación del diseño puesto en práctica.}

\section{Cuestiones a mantener y cambios a introducir.}

A la luz de los resultados, parece que la secuencia de actividades es una de las cuestiones a mantener. Alumnos y profesor coinciden en que se mejora el dinamismo de la clase, por lo que se sobrelleva mejor las dos horas de duración de la misma, sin disminuir el rendimiento. A diferencia del CM inicial, es decir, el llevado a cabo en el curso académico anterior como actividad del curso de iniciación que da acceso de la REFID, este año se ha incluido un test y postest que sirve para evaluar el aprendizaje de los alumnos. Este elemento es de especial importancia y, por tanto, otro de las cuestiones a mantener para el desarrollo del próximo curso.

Otro elemento a considerar es el mapa de contenidos. El mapa de contenido es una herramienta útil para la organización docente del profesor. No obstante, puede convertirse en una herramienta con la que, en un golpe de vista, el alumno entienda la estructura conceptual de la materia que se dispone a aprender. En este sentido, el mapa conceptual desarrollado para el CM de la asignatura es claro, aunque goza aún de cierta simplicidad, por lo que mantenerlo y seguir trabajándolo que ganar en complejidad es fundamental para el desarrollo de los siguientes ciclos de mejora. En este sentido, hacemos una propuesta para llevar a cabo en el siguiente CM, que es la de aprender a utilizar herramientas TICS destinadas a la confección de mapas conceptuales a fin de mejorar este aspecto. 


\section{Aspectos de la experiencia a incorporar en la práctica docente habitual.}

La experiencia se basa en la ampliación y mejora de la implementación llevada a cabo durante el curso de iniciación a la innovación docente realizado en el año anterior. En este curso se ha ampliado el número de temas a los que aplicar la metodología diseñada, para poco a poco cambiar l asignatura al completo. En base a los resultados obtenidos se pretende incorporar a la práctica docente o, mejor dicho, dejar fija en la misma la secuencia de actividades en la que se basa el CM.

Por otro lado, se han incluido determinadas actividades para la mejora del ciclo anterior entre las que destaca un pretest y postest de algunos conceptos básicos que forman parte de loa pilares fundamentales sobre los que se sustenta la asignatura. Esta herramienta ha permitido una evaluación objetiva del aprendizaje de los alumnos, aunque como limitación podemos destacar el bajo número de concordancia entre alumnos que hicieron el pretest y el postest. Es decir, que de 51 alumnos matriculados en la asignatura este curso, prácticamente acudieron a clase, los días que se pasaron los test, la mitad, no siendo coincidentes al 100\% los alumnos que fueron a una y otra prueba. Esto supone una limitación importante para evaluar al grupo completo y por ello proponemos usar en el próximo CM la enseñanza virtual y no hacer este tipo de test de forma presencial en clase, sino de forma virtual y obligatoria, al menos a los alumnos que asistan al $80 \%$ de las clases presenciales que son en los que se debe medir el impacto de la docencia presencial.

\section{Principios didácticos argumentados.}

Entendemos por principios didácticos el conjunto de normas o postulados que sirven como instrumento orientador para dirigir el proceso enseñanza-aprendizaje (Chi, 
Pita y Sánchez, 2011). Siguiendo el consejo de Don Finkel (2008), realicé un análisis previo sobre en qué momentos he aprendido más. Sin ninguna duda concluí que, al hacer el esfuerzo de preparar y explicar diferentes materias para otras personas, ya fuera dando clases particulares ya como docente universitario. En base a ello, me propuse dejar que hablaran los estudiantes y contaran los contenidos que previamente había extraído de un material cuidadosamente seleccionado por mi parte (Finkel, 2008). Esto supone, el primer paso para trabajar en un cambio de modelo tradicional hacia un modelo alternativo basado en la investigación como motor de aprendizaje (García Pérez, 2000b).

Vargas y Hernández (2006), han enumerado principios que pueden guiar la práctica docente y de ellos, en nuestra experimentación, se visualizan los siguientes:

3. Principio del trabajo consciente, creador, activo e independiente de los estudiantes bajo la dirección del profesor. Quizás este es el principio fundamental en el que se ha querido sustentar la experimentación, como se podrá comprobar al ver la secuencia de actividades llevadas a cabo.

4. Principio del carácter científico del contenido, puesto que el aprendizaje se sustenta en una base objetiva, real y utilizando métodos propios de las ciencias de la salud en general y de la enfermería en particular.

5. Principio de la sistematización en la conducción de la actividad docente ordenando las partes de forma que lo posterior se basa en lo anterior (Figura 3), que refleja el modus operandi del sistema sanitario en el que en un futuro desarrollarán su labor profesional los alumnos y alumnas (Vignolo, Vacarezza, Álvarez y Sosa, 2011). 
- Evitar la enfermedad

en la comunidad

- Promoción de la

Salud

Prevención

Primaria
Prevención

Secundaria

- Diagnóstico precoz
- Recuperación del

sujeto

- Evitar

complicaciones

Prevención

Terciaria

Figura 3. Actividad docente sistematizada y ordenada acorde con los niveles de prevención. Fuente: Vignolo, Vacarezza, Álvarez y Sosa, 2011.

6. Principio de la sistematización de la teoría y la práctica, dado que los casos prácticos expuesto en clase y las propias experiencias (personales o de prácticas clínicas) han permitido armonizar los conceptos teóricos con el quehacer profesional del día a día.

7. Principio de asequibilidad, que se argumenta desde la simplicidad didáctica; algo que los propios estudiantes pusieron en práctica al tener que transmitir, de forma clara, los contenidos a sus compañeros en poco tiempo.

Por otro lado, el desarrollo se ha fundamentado en crear un ambiente relajado en el que los estudiantes no se vieran amenazados por la evaluación de estas actividades, aunque sí implicados en lo que hacían, intentando con ello crear un ambiente de "aprendizaje crítico natural" (Bain, 2007). 


\section{Referencias bibliográficas}

Bain, K. (2007). Lo que hacen los mejores profesores de universidad. Valencia: Universidad de Valencia.

Cardelli J (2004). Reflexiones críticas sobre el concepto de Transposición Didáctica de Chevallard. Cuadernos de Antropología Social, 19, 49-61

Chi, A., Pita, A. y Sánchez, M. (2011). Fundamentos conceptuales y metodológicos para una enseñanza-aprendizaje desarrolladora de la disciplina Morfofisiología Humana. Educación Médica Superior, 25 (1), 3-13.

Finkel, D. (2008). Dar clase con la boca cerrada. Valencia: Universidad de Valencia.

García, F.F. (2000a). Los modelos didácticos como instrumento de análisis y de intervención en la realidad educativa. Biblio 3W. Revista Bibliográfica de Geografía y Ciencias Sociales, V (207). Recuperado de http://www. ub.edu/geocrit/b3w-207.htm

García, F.F. (2000b). Un modelo didáctico alternativo para transformar la educación: el Modelo de Investigación en la Escuela. Scripta Nova. Revista Electrónica de Geografía y Ciencias Sociales, IV (64) (15 de mayo de 2000). Recuperado de http://www.ub.edu/geocrit/sn-64.htm

Giné, N. (2009). Cómo mejorar la docencia universitaria: El punto de vista del estudiantado. Revista Complutense de Educación, 20 (1), 117-134.

González, F., Guruceaga, A.,Pozueta, E. y Porta, S. (2010). Una aproximación al conocimiento de una profesora universitaria, agente de buenas prácticas docentes, utilizando mapas conceptuales. Profesorado: Revista de Currículum y Formación del Profesorado, 14 (3), 117-130

Vargas, A. y Hernández, D. (2006). Los principios didácticos, guía segura del profesor. Pedagogía Universitaria, $11(3), 15-44$.

Vignolo, L., Vacarezza, M., Álvarez, C. y Sosa, A. (2011). Niveles de atención, de prevención y atención primaria de la salud. Archivos de Medicina Interna, XXXIII (1), 11-14. 